\title{
Tsafon
}

Revue d'études juives du Nord

$80 \mid 2020$

Varia

\section{Zeev Sternhell (1935-2020) le clerc qui n'a pas trahi}

\section{Denis Charbit}

\section{OpenEdition}

Journals

Édition électronique

URL : https://journals.openedition.org/tsafon/3336

DOI : 10.4000/tsafon.3336

ISSN : 2609-6420

\section{Éditeur}

Association Jean-Marie Delmaire

\section{Édition imprimée}

Date de publication : 1 décembre 2020

Pagination : 115-136

ISSN : 1149-6630

\section{Référence électronique}

Denis Charbit, «Zeev Sternhell (1935-2020) le clerc qui n'a pas trahi », Tsafon [En ligne], 80 | 2020, mis en ligne le 01 décembre 2020, consulté le 23 juin 2021. URL : http://journals.openedition.org/tsafon/ 3336 ; DOI : https://doi.org/10.4000/tsafon.3336 


\section{Zeev Sternhell (1935-2020) le clerc qui n'a pas trahi}

Denis Charbit*

Les historiens ont en commun le souci de reconstituer une époque, un milieu, des hommes et des idées, au plus près de la vérité. Une fois ce consensus acquis, ils se distinguent les uns des autres quel que soit le critère sélectionné. Si l'on retient la période historique sur laquelle ils travaillent, on discernera, par exemple, les spécialistes de l'Antiquité, les médiévistes, les vingtièmistes. Le critère peut être relatif au lieu: européanistes, africanistes, orientalistes; ou bien à l'approche sélectionnée: certains font de l'histoire sociale ou de l'histoire économique, d'autres de l'histoire culturelle ou intellectuelle, de l'histoire militaire ou diplomatique, religieuse ou politique. Quelle que soit la typologie proposée, théorique ou méthodologique, les historiens se répartissent équitablement entre les différentes catégories établies à cet effet.

Il est une typologie pourtant dans laquelle une majorité d'historiens se retrouve dans une catégorie, tandis qu'une poignée seulement se range dans la seconde. Si la curiosité est identique pour tous, la plupart des historiens confessent sans hésiter une inclination, une empathie, voire une admiration pour les hommes ou les idées qu'ils placent au cœur de leur recherche. Une minorité, en revanche, déclare sans détour qu'ils n'éprouvent aucune sympathie pour leurs héros qui font plutôt figure, à leurs yeux, d'anti-héros. Ils éprouvent, dans certains cas, de la répulsion

\footnotetext{
* Département de sociologie, science politique et communication. The Open University of Israel (Ra'anana).
} 
pour ceux dont ils retracent l'action ou les idées. Zeev Sternhell (19352020) a fait résolument partie de cette seconde catégorie, à laquelle adhèrent également les historiens du fascisme, du stalinisme, du nazisme, du racisme, du colonialisme et des génocides.

Les pages qui suivent sont un essai de synthèse d'une œuvre interrompue, mais non inachevée, avec la disparition de l'historien israélien auquel nous souhaitons ainsi rendre hommage. Outre cette tentative de dégager les principaux axes de sa recherche, puis d'étudier les causes de sa réception controversée, ce texte, publié dans une des dernières revues d'études juives qui existent encore dans le monde francophone, souhaite répondre à deux questions qui relèvent des études juives et des études israéliennes et ont émergé de cet examen auquel nous avons procédé.

Spécialiste du fascisme français et de la nébuleuse de penseurs et de leaders, de ligues et de cercles violement hostiles à la République et déterminés à la renverser, Sternhell, dans cette plongée en eaux troubles, s'est inévitablement heurté à l'hydre de l'antisémitisme sans pour autant avoir été considéré, ou s'être considéré lui-même, comme un historien de l'antisémitisme. Aussi nous souhaiterions évaluer la place que cette passion funeste occupe dans son œuvre, autrement dit, l'importance que Sternhell lui a conférée dans la vision du monde propre à cette tendance politique qu'il a étudiée en profondeur. Notre deuxième interrogation, moins évidente encore, nous entraînera du côté d'un de ses livres, intitulé dans sa version française Aux origines d'Israël, et qui tranche avec tous les autres puisqu'il est le seul à traiter du sionisme et du yishouv. Sternhell n'était pas de ces historiens éclectiques qui étendent d'un livre à l'autre leurs domaines de compétence ${ }^{1}$. Peut-on cependant établir un lien, dont il faudra préciser et nuancer la nature, entre Les Origines françaises du fascisme (le sous-titre de La Droite révolutionnaire) et les Aux origines d'Israël, publié, en hébreu sous le titre, Binyane Ouma o Tikkoun Hevra [Nation-Building ou Réforme de la sociétée $]^{2}$ ? Ou bien convient-il de n'y voir que l'appendice scientifique d'un très vaste corpus

\footnotetext{
${ }^{1}$ Notons, à titre d'exemple, le cas de Marc Ferro, qui fut spécialiste de la Première Guerre mondiale et de la Révolution russe, mais aussi de cinéma et histoire, de l'enseignement de l'histoire et publia un ouvrage sur Pétain de près de 800 pages exclusivement consacrées à son exercice du pouvoir à Vichy de 1940 à 1944, cf. Marc Ferro, Pétain, Paris, Fayard, 1987.

${ }^{2}$ Il va de soi que cela ne saurait être une quelconque parenté entre sionisme et fascisme que seuls les adversaires acharnés d'Israël pourraient concevoir, encore que sous leur plume trempée au vitriol, la comparaison - que dis-je ? - l'identification du sionisme au nazisme et à l'apartheid leur paraît beaucoup plus séduisante.
} 
constitué de chroniques et d'articles de presse qu'il a écrit en intellectuel soucieux de participer activement au débat public, en militant de la gauche israélienne qu'il a observée de l'intérieur sur plus d'un demisiècle, depuis la fin des années 1960 et jusqu'à ses derniers jours ?

Avant d'apporter une réponse à ces deux questions, tentons de dégager ce qui a fait l'originalité et l'audace de ses thèses sur le fascisme au point de susciter une telle polémique.

\section{La quête des origines}

Sternhell a très vite saisi qu'expliquer la montée du fascisme (ou du nazisme) par les contradictions du capitalisme était largement insuffisant. Pour être esthétiquement impressionnant, le célèbre montage photographique de John Heartfield, Der Sinn des Hitlergrusses [Le sens $d u$ salut hitlérien], montrant la main brandie vers le haut et tendue en l'air du Führer en quête de quelques billets froissés et jetés par un représentant $\mathrm{du}$ capitalisme international, était un argument peu convaincant d'un point de vue historique et, en vérité, dérisoire. Non que les représentants du capital n'aient pas cru bon soutenir Hitler au moment où il accédait au pouvoir ${ }^{3}$, mais Sternhell ne croyait guère qu'une telle rupture dans la civilisation pouvait être un accident, une contingence, le fruit d'une alliance ad hoc qui aurait bouleversé à ce point le cours de l'histoire. La conjoncture, la coïncidence, le hasard peuvent avoir un certain impact, mais ils n'entraînent pas à eux seuls un continent dans l'abîme, tels des dominos qui s'effondrent l'un après l'autre, pays par pays. Pour renverser la monarchie absolue et l'Église triomphante, il avait fallu qu'apparaissent, sur le plan intellectuel et idéologique, la philosophie des Lumières puis, au niveau politique, la Révolution française prompte à donner à l'Ancien régime le coup de grâce. Autrement dit, l'action avait été précédée d'un bouillonnement d'idées qui avait préparé le terrain pour les forces sociales et politiques capables de s'emparer de ce discours nouveau et d'en tirer des mots d'ordre audibles. Suivant une démarche analogue, Sternhell ne pouvait admettre que le fascisme, le nazisme et le communisme étaient les enfants bâtards et déformés de la même mère: la Première Guerre mondiale. La

\footnotetext{
${ }^{3}$ Charles Bettelheim a livré une explication de ce type dans son livre L'Économie allemande sous le nazisme, Paris, Marcel Rivière, 1945. Très récemment, empruntant la voie romanesque, Éric Vuillard a montré la complicité criminelle des élites économiques avec Hitler dans son livre distingué par le prix Goncourt L'ordre du jour, Arles, Actes Sud, 2018.
} 
chronologie, pour indispensable qu'elle soit, ne pouvait tenir lieu d'explication historique. Qu'elle nourrisse le sens commun, sans aucun doute : comment n'établirait-on guère un lien de cause à effet entre la boucherie de 1914-1918 et un défi révolutionnaire de grande ampleur, de gauche et de droite, la Révolution russe en 1917 et la marche sur Rome en 1922 ? Sternhell pouvait bien admettre que la Grande Guerre ait constitué un catalyseur offrant au communisme et au fascisme une opportunité dont leurs leaders respectifs n'allaient pas manquer de se saisir, il ne crut pas devoir l'identifier, du fait de l'ampleur du traumatisme, comme la cause profonde de leur émergence. Sternhell, à sa manière, était sensible à la longue durée. Si l'individu, la démocratie et la raison avaient été balayés, c'est bien qu'une triade de substitution élaborée le temps d'une génération, voire deux, était mûre pour prétendre la remplacer : la race, la nation, le leader s'étaient cristallisés en fer de lance de nouveaux régimes qui se sont maintenus grâce à l'usage de la Terreur.

Il faut bien voir toutefois que ce n'est pas l'apogée du fascisme ou du nazisme qui a retenu l'attention de Sternhell, encore moins leur chute. Ce n'est pas non plus leur ascension irrésistible qu'il a tenté d'explorer pour en relater les étapes, c'est leur naissance qu'il est allé examiner de près. Aussi est-ce toujours en amont que Sternhell pose son scalpel. D'où sa propension à dévoiler les origines plutôt qu'à tirer les conséquences.

C'est par ce point de vue soucieux et curieux de l'antériorité qu'il a pu se rendre compte que les leaders n'ont rien pensé, conçu et inventé, ou si peu. Ils n'ont eu qu'à ramasser, qu'à rassembler ce qu'avaient élaboré avant-guerre, de revue en revue, de journal en journal, des écrivains, des penseurs, des théoriciens. À la différence de ses collègues qui se sont spécialisés, comme lui, dans le mal en politique, Zeev Sternhell n'a guère placé les hommes politiques au cœur de sa recherche. En s'attaquant aux clercs, plutôt qu'aux hommes d'action, Sternhell a rétabli l'importance des idées dans l'histoire, ces idées que Marx avait dénigrées en faisant d'elles une superstructure, un reflet, une écume superficielle comparée aux rapports de production et à la place qu'y occupent les individus et les groupes sociaux selon qu'ils appartiennent à la bourgeoisie ou au prolétariat. Le postulat de Sternhell était aussi simple qu'audacieux dans un contexte historiographique dominé dans les années 1970 par l'histoire économique et sociale : ce sont les idées qui mènent le monde. À cet égard, même si Marx ne fut pas une source féconde pour son travail d'historien, il en a néanmoins retenu quelque chose de la structure binaire 
qu'il avait repérée : l'histoire n'était peut-être pas pour lui l'affrontement de deux classes, elle était sans aucun doute le choc entre deux idées, et pour ce qui est de l'histoire contemporaine, la bataille sans merci à laquelle s'étaient livrées depuis le XVIII ${ }^{\mathrm{e}}$ siècle les Lumières et les antiLumières. Car, pour que s'impose ce nouvel ordre, il fallait bien avoir fait des Lumières table rase. Pour imposer des idées neuves, il importait de broyer les anciennes, de balayer l'édifice progressif et imparfait des démocraties libérales et parlementaires accrochées à la triade républicaine que leurs adversaires avaient vidée de son sens : la liberté, l'égalité, la fraternité n'était plus que celle de la race supérieure, celle du Volk, précipitant à la trappe deux siècles d'élaboration intellectuelle dont la méthode était rationnelle sinon rationaliste et la finalité universelle. Ce basculement d'une société dans l'abîme et dans le crime n'était pas réductible au charisme d'un leader, à sa capacité de tirer parti avec habileté des circonstances. Il exigeait une préparation des cœurs, une mobilisation des idées jusqu'à l'endoctrinement, une exaltation des instincts grégaires, une démission des clercs pour signer la faillite des démocraties. Autrement dit, le fascisme n'était pas, dans l'esprit de Sternhell, un patchwork, un ramassis d'idées orphelines, un bric-à-brac idéologique, toutes sortes de définitions destinées à en souligner le caractère spontané, improvisé, éclectique, décousu, ô combien réducteur. Mais en braquant son attention sur les clercs, Sternhell avait couru le risque de déchaîner les passions. S'il s'était occupé, par exemple, de Pétain, l'historien israélien n'aurait guère suscité de controverse et ne se serait pas fait d'adversaires. Alors que les leaders peuvent difficilement se prévaloir d'historiens prompts à défendre leur bilan, les meneurs d'idées, les manieurs d'idées, justement parce qu'ils sont romanciers, artistes, philosophes, penseurs à distance de l'action, suscitent souvent engouement et admiration pour leur œuvre. En les plaçant non au centre d'une histoire littéraire ou intellectuelle, mais dans l'intersection entre histoire des idées et histoire politique, Sternhell s'est exposé aux foudres de ceux qui tenaient coûte que coûte à conserver de leur auteur ou de leur penseur de prédilection une image d'Épinal. Même s'ils s'étaient immiscés en politique, puis égarés, leur engagement est toujours apparu, aux yeux de leurs admirateurs, une étape sans importance, un incident de parcours. Rien d'un engagement primordial, pas même d'un engagement second, tout au plus un engagement secondaire. Sternhell a renversé la hiérarchie, plaçant l'engagement politique de ses écrivains et penseurs au cœur de sa recherche. 


\section{Un regard neuf sur le fascisme}

Ce n'est pas de Barrès l'écrivain que Sternhell a dressé le portrait, mais du penseur et tribun politique ${ }^{4}$. Non que cet aspect ait été ignoré ou occulté, il était, en général, réduit à sa dimension strictement biographique et perçu comme dépourvu de tout impact, et dont il ne resterait plus de trace, comparé aux chefs d'œuvre du grand homme. Qui, hors Sternhell, pouvait dénicher dans une publication de province obscure et étalée sur quelques numéros, Le Courrier de l'Est, la conjonction sociale, nationaliste et antisémite qui faisait de Barrès le chantre d'une idéologie d'un type nouveau ? En outre, Barrès continuait d'exercer un ascendant littéraire qui perdurait encore dans les années 1960 comme en témoigne le volume de la collection «Écrivains de toujours » aux éditions du Seuil qui lui fut consacré, aussi étrange que cela puisse paraître, par Jean-Marie Domenach, alors rédacteur-en-chef de la revue personnaliste de gauche, Esprit.

Il n'était pas encore clair pour lecteur de ce premier livre tiré de sa thèse que Sternhell poursuivrait son enquête jusqu'à Vichy. Après coup, on peut discerner dans l'ensemble de son œuvre une trilogie française dont le premier volume était le Maurice Barrès et le nationalisme français paru en 1972, le second volume La Droite révolutionnaire, publié en 1978 et le troisième volume, Ni Droite ni gauche, qui date de 1983. Cette trilogie épouse bien une ligne chronologique puisque le Barrès s'arrête en 1914, le second reprend la même période, mais élargit la recherche du cas Barrès aux nébuleuses de la droite antirépublicaine surgie aux lendemains de la crise Boulanger et de l'affaire Dreyfus autour desquelles gravitent, outre Maurras et Barrès déjà cité, de nouveaux protagonistes: Jules Soury, Jules Guérin, Griffuelhes, Lagardelle, Edouard Berth. Autant dire qu'ils étaient totalement inconnus lorsque Sternhell s'en est emparé. Ce n'est pas qu'ils soient devenus célèbres depuis. Ce n'est pas tant les avoir exhumés qui importe, mais d'avoir montré qu'ils disposaient d'une large audience et influence. Et, plus que leur impact encore, l'apport fondamental de Sternhell est d'avoir révélé que ces théoriciens de droite n'étaient pas de fieffés réactionnaires, mais des figures plus radicales encore que les pères de la Contre-Révolution

\footnotetext{
${ }^{4}$ Zeev Sternhell, Maurice Barrès et le nationalisme français, Paris, Armand Colin et Presses de la Fondation nationale des Sciences politiques, 1972 ( $1^{\text {ère }}$ édition), 2000 ( $2^{\text {ème }}$ édition, Fayard).
} 
du début du XIX ${ }^{\mathrm{e}}$ siècle, Joseph de Maistre et Louis de Bonald. Dans leur quête d'alliés et d'alliance, ce n'est pas du côté de la vieille France catholique, intransigeante et patriote qu'ils se sont tournés, mais du côté des ouvriers et de la gauche radicale. Ils ne se sont pas réclamés d'un conservatisme ultra, mais de la révolution, non qu'ils aient absorbé le patrimoine de 1789, ils entendaient bien mettre à bas la République et transformer de fond en comble les institutions. Pour assurer cette circulation, ce n'est pas la contribution de Maurras qui joua un rôle de premier plan, mais celle de Georges Sorel. La droite révolutionnaire ne fut pas sans attirer à elle des militants provenant de l'anarchosyndicalisme.

Lors de sa parution en 1978, La Droite révolutionnaire s'est pleinement inscrit dans la vague iconoclaste de la génération montante qui déboulonnait, une à une, les statues du Commandeur ${ }^{5}$. Mai 68 avait jailli, de Gaulle était mort, et la contestation soufflait dru sur les thèses dogmatiques à force d'avoir été dominantes. François Furet portait un coup à la vulgate marxiste en restituant l'importance de l'historiographie libérale et la pertinence de la parenthèse réformiste de la Révolution, de trop courte durée, étouffée par la Terreur ${ }^{6}$; l'historien américain Robert Paxton avait pulvérisé le mythe d'une alliance implicite entre de Gaulle et Pétain, l'épée et le bouclier, la Résistance et Vichy, élaboré par Robert Aron dans son Histoire de Vichy à des fins de réconciliation nationale ${ }^{7}$. S'appuyant sur les archives allemandes notamment, il avait magistralement démontré que le gouvernement de Vichy ne s'était pas plié au diktat allemand, mais avait devancé même les instructions de l'occupant en matière d'antisémitisme en promulguant le Statut des Juifs, trois mois à peine après la proclamation de la Révolution nationale.

En prenant pour cible Albert Soboul et l'historiographie marxiste de la Révolution française, Furet s'attaquait déjà à un colosse aux pieds d'argile tandis que la thèse de Robert Aron mise en pièces par Paxton s'écroulait d'autant plus que le premier n'était pas un historien de formation. Seul Sternhell faisait d'une pierre deux coups : d'un côté, en

\footnotetext{
${ }^{5}$ Zeev Sternhell, La Droite révolutionnaire 1865-1914. Les origines françaises du fascisme, Paris, Seuil, coll. "L'Univers historique », 1978 ( $1^{\text {ere }}$ édition), 1997 ( $2^{\text {ème }}$ édition, Gallimard), 2000 ( $3^{\text {ème }}$ édition, Fayard).

${ }^{6}$ François Furet et Denis Richet, La Révolution française, Paris, Hachette, 1965 ; François Furet, Penser la Révolution française, Paris, Gallimard, coll. «Bibliothèque des Histoires », 1978.

${ }^{7}$ Robert Paxton, La France de Vichy 1940-1944, Paris, Seuil, 1973 ; Robert Aron, Histoire de Vichy, Paris, Fayard, coll. « Les Grandes études contemporaines », 1954.
} 
qualifiant de "révolutionnaire » une droite qui n'était pas encore définie comme l'extrême-droite, il faisait d'un oxymore apparent un syntagme crédible ; par ce titre, La Droite révolutionnaire, il secouait une thèse devenue un classique du genre, sur laquelle René Rémond avait établi sa réputation : La Droite en France ${ }^{8}$. Quand bien même Sternhell évitait de crier haro sur le livre de Rémond, il n'en restait pas moins vrai que sa célèbre typologie distinguant entre le légitimisme, l'orléanisme et le bonapartisme, pour pertinente qu'elle demeurait pour quiconque s'intéresse à la droite, s'avérait néanmoins incomplète. Rémond avait nié cette tendance ou, mieux encore, il ne l'avait pas repérée. Sternhell la faisait surgir ex nihilo, textes à l'appui, archives dépouillées de fond en comble. La démonstration était imparable. Désormais, il n'était plus possible de parler de la droite française au $\mathrm{XX}^{\mathrm{e}}$ siècle sans cette composante révolutionnaire exhumée et dépoussiérée par Sternhell.

\section{Vichy, la fin de la parenthèse}

En amont, non en aval; des clercs plutôt que des hommes politiques ; une conception du monde plutôt que des actes ; telles sont les différentes facettes de la démarche de Sternhell à laquelle il faut encore ajouter l'ultime coup de grâce qui lui vaudra tant d'incompréhension et de polémique et autour duquel tout historien de la France tourne sans cesse : l'interprétation de la défaite de 1940 et le régime de Vichy. D'autres que lui ont tiré au clair la faillite des élites économiques, des élites politiques, celle des fonctionnaires et des juges. Marc Bloch en a livré le témoignage accablant dans son Étrange défaite. Sternhell a ausculté cette étrange défaite avant la défaite elle-même et en est ressorti avec une conclusion péremptoire : l'étrange défaite n'était pas si étrange que cela. Elle n'était pas réductible à une erreur de stratégie commise par des généraux qui se sont trompés de guerre, pas plus qu'à une tendance relevant de la psychologie collective commune à un grand nombre de Français qui s'étaient juré que 1914-1918 serait la « der des ders ». L'étrange défaite, pour Sternhell, ne s'épuisait guère dans le fracas et le verdict des armes, mais dans la reddition des âmes: un cessez-le-feu n'avait rien de déshonorant, c'est la liquidation du régime consentie par une Assemblée et un Sénat réunis offrant les pleins pouvoirs au Maréchal Pétain pour substituer à la Révolution française la Révolution nationale, qui l'était.

\footnotetext{
${ }^{8}$ René Rémond, La Droite en France, Paris, Aubier-Montaigne, 1954. Réédité en 1963, c'est en 1982 que le titre est passé au pluriel : Les Droites en France.
} 
Procédant à l'archéologie de cette substitution de régime, Sternhell n'eut pas la faiblesse et la complaisance de penser que le triomphe de Vichy était dû aux événements, à la conjonction fatale d'une défaite militaire et d'une faillite politique. Que les élections qui s'étaient déroulées dans l'entre-deux-guerres n'aient jamais porté au pouvoir ces fossoyeurs de la démocratie, qu'elles aient même abouti à confier à un homme politique d'origine juive, Léon Blum, le soin de conduire un gouvernement de Front populaire à l'origine des réformes les plus sociales, les plus généreuses et les plus étendues qu'ait jamais connu le pays depuis la Révolution française, n'a guère ébranlé l'intuition profonde de Sternhell. Aussi singulières et inattendues qu'aient été les circonstances, tout était prêt en France pour y accueillir Vichy : les hommes et l'état d'esprit. Ces hommes ne faisaient pas cavalier seul, ils étaient les maillons d'une nouvelle culture politique qui s'était déclinée avec sa grammaire et son vocabulaire, ses mots-clés et son jargon depuis un demi-siècle.

Ce fut là l'audace de trop et elle ne lui fut guère pardonnée : Sternhell ne trouvait pas étrange la défaite de 1940, il estimait surtout qu'elle n'était pas étrangère à la France, en dépit de Zola et de son fameux $J^{\prime} a c c u s e$, en dépit de l'acquittement de Dreyfus et de sa réhabilitation, en dépit de la victoire de coalition dreyfusarde, de la séparation des Églises et de l'État, de la liquidation au sein de la gauche de l'antisémitisme passé, dès lors, avec armes et bagages, du côté de ce que Sternhell avait appelé, réceptif à l'oxymore, la droite révolutionnaire. Toutes ces étapes et tous ces événements ont généré et scellé le mythe d'une République qui, après avoir vacillé un temps, s'est aussitôt ressaisie pour conjurer le retour de la bête. Or, la bête est revenue, et celle-ci n'a pas eu à franchir les frontières dans les fourgons de l'étranger; il n'a pas été nécessaire de l'importer d'Allemagne ou d'Italie, il a suffi de la rapatrier pour qu'elle puisse servir, à l'intérieur, le régime de Vichy.

En se proposant d'ausculter les deux décennies consécutives à la Première Guerre mondiale, comme il l'avait fait dans le livre précédent pour les années antérieures à la guerre, Sternhell récidivait ${ }^{9}$. Il n'eut pas, cette fois, à exhumer des noms et à dépoussiérer des volumes de revues tombées dans l'oubli. Les protagonistes étaient relativement familiers, certains vivaient encore (Alfred Fabre-Luce, Bertrand de Jouvenel, Thierry Maulnier, Jean de Fabrègues) ou venaient tout juste de

\footnotetext{
9 Zeev Sternhell, Ni droite ni gauche. L'idéologie fasciste en France. Paris, Seuil 1983 ( $1^{\text {ère }}$ édition), 1987 ( $2^{\text {ème }}$ édition, Complexe), 2000 ( $3^{\text {eme }}$ édition, Fayard), 2012 ( $4^{\text {ème }}$ édition, Gallimard, coll. « Folio-Histoire »).
} 
disparaitre (Gaston Bergery, Emmanuel Berl). Cette effervescence intellectuelle avait d'ailleurs fait l'objet d'une première synthèse. Publié aux éditions du Seuil dans la foulée de mai 68, le livre avait été titré : Les Non-conformistes des années trente ${ }^{10}$. Mais alors que son auteur, JeanLouis Loubet Del Bayle, s'était montré bienveillant, curieux et admiratif pour cette pépinière de talents et d'esprits critiques, voilà que Sternhell révisa de fond en comble cet euphémisme laudateur pour en tirer un jugement on ne peut plus sévère sur ces non-conformistes dont un bon nombre allait sombrer dans la collaboration. Rejetant les étiquettes parlementaires, « ni droite ni gauche», comme Sternhell les a caractérisés, ils avaient dénigré, bien avant la défaite, l'épuisement de la démocratie libérale qu'ils tenaient pour avachie, paralysée, et tout bonnement incapable d'insuffler la vigueur aux élites comme aux masses, qui avait rénové l'Italie et l'Allemagne. Ce sont eux qui ont préparé une alternative à la démocratie parlementaire et préfiguré l'esprit de Vichy et sa Révolution nationale.

Alors que Sternhell avait, sans mot dire, mis en cause le livre de son collègue René Rémond, il plaça, cette fois, dans sa ligne de mire, et dans les toutes dernières pages du livre, la figure charismatique d'Emmanuel Mounier. Le fondateur d'Esprit avait inspiré toute une génération d'hommes politiques, de hauts fonctionnaires et d'historiens parmi lesquels les éditeurs même des livres de Sternhell : Jacques Julliard et Michel Winock. Il n'avait pas été pourtant le premier à mettre Mounier à l'épreuve. Deux ans avant la parution de Ni droite ni gauche, dans un essai aux accents polémiques volontairement assumés, Bernard-Henri Lévy avait dénoncé tout un pan nationaliste et particulariste de l'idéologie française, pour reprendre le titre éponyme de son livre, qu'il prenait soin de ne pas circonscrire à Barrès, Maurras, Brasillach et Drieu La Rochelle pour y inclure Péguy et Mounier ${ }^{11}$. Bernard-Henri Lévy fut violemment pris à partie par Jean-Marie Domenach ${ }^{12}$. Aussi était-il prévisible que les disciples d'Esprit n'allaient pas rester de marbre devant le brûlot de Sternhell. Ils vécurent cette nouvelle épreuve comme un harcèlement face auquel ils se trouvaient démunis, car il leur parut aisé de démonter l'autorité de Bernard-Henri Lévy. Celui-ci pouvait bien être un «nouveau philosophe », il n'était pas un historien capable d'interpréter des sources

\footnotetext{
10 Jean-Louis Loubet Del Bayle, Les Non-conformistes des années trente, Paris, Seuil, 1969 ( $1^{\mathrm{e}}$ édition), 2001 ( $2^{\mathrm{e}}$ édition).

11 Bernard-Henri Lévy, L'Idéologie française, Paris, Grasset, coll. « Figures », 1981.

${ }^{12}$ Voir le numéro de la revue Esprit n ${ }^{\circ}$, Mai 1981, p. 2-42.
} 
primaires et des archives remontant aux années 1930 et 1940 sans tomber dans la polémique, le pamphlet et l'anachronisme. Sur le terrain du savoir et sur sa pratique du métier d'historien, Sternhell était inattaquable. Sternhell fut témoin alors d'un retournement de situation sans précédent : alors que la publication de La Droite révolutionnaire avait été encouragée et encensée par Julliard et Winock, qui avaient tous deux fait leurs premières armes critiques à Esprit, voilà qu'avec ce second livre paru dans la même collection, Sternhell était voué par les mêmes aux gémonies $^{13}$. Une fois de plus, fidèle à son point de vue, Sternhell ne s'en était pas pris aux hommes politiques et aux fonctionnaires du régime Pétain, Laval, Vallat, Darquier de Pellepoix, Jardin ou Bousquet - mais aux intellectuels dont il scrutait le comportement et les déclarations avec gravité et sévérité. Même s'il expliquait bien que Mounier avait fini par quitter Uriage, qu'il était entré dans la clandestinité puis fut arrêté et incarcéré, Sternhell, fidèle à sa façon de penser, refusa de concéder que la parution de dix numéros de la revue de novembre 1940 (n94) à août 1941 ( $\left.\mathrm{n}^{\circ} 103\right)$ sous le contrôle et la censure de Vichy, au lieu de la saborder, avait été dans le parcours de Mounier un moment d'égarement, un passage à vide, une parenthèse, un mauvais calcul ou quelque motif que ce soit qui puisse être traité avec indulgence. Cette bienveillance posthume était pour lui signe de complaisance. Il persistait à tenir ce flirt avec la Révolution nationale non pour une adaptation opportuniste aux circonstances consécutives à l'Armistice, mais pour une collusion négative et une affinité entre Mounier et le régime de Vichy concernant la démocratie parlementaire et l'individualisme bourgeois. Pour Sternhell, le ver était dans le fruit. Dans les colonnes d'Esprit des années trente, en dépit du soutien au Front populaire, de la solidarité avec la République espagnole aux prises avec le fascisme, et du rejet de l'esprit munichois, Mounier n'avait cessé d'adresser à la démocratie parlementaire et à l'esprit bourgeois un réquisitoire sans concession qui tenait de l'aversion. N'étant ni communiste ni socialiste, encore moins un «rad-soc», Mounier était donc un adversaire du libéralisme et du marxisme, ce qui le

\footnotetext{
${ }^{13}$ Sur la polémique soulevée par les livres de Sternhell, cf. Antonio Costa Pinto, " Fascist Ideology Revisited: Zeev Sternhell, and His Critics», European History Quarterly, vol. 16, October 1986, p. 465-483 ; Robert Wohl, «French Fascism, Both Right and Left : Reflections on the Sternhell Controversy », Journal of Modern History, vol. 63, March 1991, p. 91-98. On peut renvoyer, en français, à l'entretien suivant: Marco Diani et Michela Nacci, «Fascisme: idéologie française et intellectuels européens. Entretien avec Zeev Sternhell», Contemporary French Civilization, vol. XIV n ${ }^{\circ}$, Winter 1990, p. 49-62.
} 
rapprochait du fascisme et de Vichy dont il était devenu complice en cela même qu'il avait endossé le rôle de fossoyeur de la démocratie et de la III $^{\text {e }}$ République. La thèse était avancée sans nuance, avec une force de conviction qui secouait violemment celui que Julliard et Winock tenaient pour leur maître à penser. Pour Sternhell, il aurait suffi que les thuriféraires d'Emmanuel Mounier avouent la faute commise par leur aîné plutôt que de persister dans la dénégation quarante ans après les faits et de s'ériger en gardiens du culte.

Historien des idées, Sternhell a pris les idées au sérieux. Un intellectuel n'y adhère pas à cause d'un événement ou d'un caprice. Elles ne sont pas le produit de circonstances fortuites : celles-ci peuvent les faire passer du stade théorique à une réalité politique inscrite dans un régime particulier, elles ne peuvent en être la cause profonde. Le fascisme et le communisme triomphent dans le sillage de la guerre de 1914, mais leurs principes et leurs revendications sont antérieures. Personne n'en doute à propos du communisme grâce à la figure tutélaire de Marx ; pour le fascisme, Sternhell a bien montré que son avènement politique au début des années 1920 en Italie est précédé d'une fertilité intellectuelle spectaculaire qui s'est déroulée avant-guerre. Cette percée sternhellienne s'accompagne d'une autre révélation tout aussi explosive : dans ce fascisme de papier, la part des penseurs français fut capitale. $\mathrm{Ni}$ les D'Annunzio ni les Marinetti et son groupe futuriste n'ont été les pionniers du fascisme, tout au plus des propagateurs. C'est en France, avec un Georges Sorel notamment, que le terreau s'est constitué. Bref, il n'y eut pas qu'un fascisme à la française ou un fascisme en France, il y eut un fascisme de France, un fascisme français.

La controverse Sternhell était née, elle ne s'est jamais éteinte depuis. Elle ne porte pas sur les faits ou les discours, mais sur leur interprétation et leur appréciation. Sternhell voyait dans cette cabale dirigée contre lui l'effet d'une blessure narcissique que ses excompagnons croyaient soigner en attaquant le messager. Il crut bon d'avancer que leur volte-face était due à des calculs, des affaires d'intérêts et de carrière, ce qui déplaçait inutilement la controverse légitime du côté de la polémique ad hominem.

Il semble pourtant que, contrairement aux allégations de Sternhell, le regard porté sur Vichy se soit considérablement modifié : qui pense encore en France qu'il n'était qu'une parenthèse, hormis quelques historiens qui ont quelque scrupule à considérer le gouvernement de Vichy comme un régime fasciste? 
Cette trilogie française, augmentée d'un volume sur la Naissance de l'idéologie fasciste, fut complétée par une somme dont le cadre spatial et temporel dépassait la France de la Troisième République : le regard s'étendait à l'Europe, " $d u$ XVIIIe siècle à la guerre froide », comme le sous-titre du livre l'indique. Sternhell remonte jusqu'à Edmond Burke, Herder et Joseph de Maistre et joint à ce grand cercle des penseurs disparus les figures prévisibles de Taine et de Spengler, et celles, plus inattendues, d'Ernest Renan et d'Isaiah Berlin. Sternhell embrasse deux siècles de réflexion qu'il récapitule, non par un contenu positif, mais par une déclaration de guerre : Les anti-Lumières ${ }^{14}$.

\section{La place de l'antisémitisme}

Si Sternhell ne s'est jamais considéré comme un historien de l'antisémitisme, il reste que son archéologie du fascisme et de la droite révolutionnaire l'a confronté à la tâche d'apprécier et d'évaluer qualitativement sa place au cœur de cette famille politique résolument hostile aux Lumières. L'émancipation des juifs, votée en 1791, est apparue, à juste titre, comme la réforme emblématique de l'esprit universaliste découlant de la philosophie politique des Lumières et mise en œuvre par la Révolution française, nonobstant l'ambiguïté d'un Kant et d'un Voltaire envers le judaïsme et les juifs ${ }^{15}$. Aussi Sternhell note bien que la droite révolutionnaire, faisant flèche de tout bois contre la démocratie parlementaire et libérale, eut à cœur de hisser l'antisémitisme au panthéon de son idéologie. Quiconque voulait abattre la démocratie et la république était nécessairement antisémite, et celui qui ne l'était pas faisait figure d'exception. Cependant, Sternhell se démarque des historiens de l'antisémitisme sur l'identité de la cause et de l'effet. Pour l'historien du fascisme, la haine de la démocratie libérale précède et aboutit à l'antisémitisme, tandis que pour l'historien de l'antisémitisme, la haine des juifs, primaire et première, conduit au rejet de la démocratie libérale coupable d'avoir inclus les juifs dans la cité.

\footnotetext{
${ }^{14}$ Zeev Sternhell, Les anti-Lumières. Du XVIIIe siècle à la guerre froide, Paris, Fayard, coll. «L'espace du politique », 2006 ( $1^{\text {ere }}$ édition), 2010 ( $2^{\text {eme }}$ édition, Gallimard, coll. « Folio-Histoire »).

${ }^{15}$ On citera ici les ouvrages qui ont inauguré l'ère du soupçon critique envers les Lumières, lesquelles n'ont pas toujours manifesté le versant inclusif qui leur est attaché : Léon Poliakov, Histoire de l'antisémitisme vol. 3: De Voltaire à Wagner, Paris, Calmann-Lévy, coll. "Liberté de l'esprit», 1968 ; Arthur Hertzberg, The French Enlightenment and the Jews. The Origins of Modern Anti-Semitism, New York, Columbia University Press and Jewish Publication Society of America, 1968.
} 
Les deux premiers livres de Sternhell accordent une place significative à l'antisémitisme. Dans le livre sur Barrès, le chapitre V consacré aux " Premiers éléments d'une doctrine nationaliste » examine, tour à tour, "La question nationale », "Le socialisme nationaliste », enfin, «L'antisémitisme social ». Barrès, explique Sternhell, ne s'est pas découvert antisémite, place des Invalides, lors de la dégradation du capitaine Dreyfus. Il avait intégré cette passion politique dix ans plus tôt, avec l'affaire Boulanger. Au-delà du cas Barrès, Sternhell montre que l'antisémitisme devient le ciment interclassiste par excellence pour fédérer le prolétariat, les couches populaires, les classes moyennes et les élites issues de la bourgeoisie et de la noblesse et rassembler France des terroirs et France urbaine, révolutionnaires et conservateurs, catholiques et libres penseurs (mais pas les protestants et les francs-maçons). La Droite révolutionnaire consacre une part plus substantielle encore à l'antisémitisme, pas moins de deux chapitres conséquents sur les neuf que comporte le livre $^{16}$. Cet antisémitisme n'est plus un adjuvant pratique et instrumental qui sert de liant, il devient un ingrédient indispensable étroitement lié au rejet de la démocratie. Ce qui en ressort est bien la plasticité de l'antisémitisme dont toutes les tendances peuvent faire bon usage, et parfois un usage massif aux deux extrémités de l'échiquier politique et idéologique.

Cette dynamique ascendante de l'antisémitisme dans les deux premiers ouvrages est moins apparente dans les trois derniers. Dans Naissance de l'idéologie fasciste, Sternhell montre, sur le plan individuel, le virage antisémite effectué par Georges Sorel à partir de $1910^{17}$. Plus généralement, il fait du déterminisme racial et biologique le critère de distinction entre nazisme et fascisme : «les deux idéologies, les deux mouvements et les deux régimes possèdent des points communs, [...] mais ils diffèrent sur une question fondamentale : la pierre de touche du national-socialisme allemand est le déterminisme biologique [...] $\mathrm{Si}$ Robert Brasillach professe un antisémitisme très proche de celui du nazisme, le Faisceau de Georges Valois en est totalement dépourvu ${ }^{18}{ }^{\text {. }}$

\footnotetext{
${ }^{16}$ Citons-les tels que Sternhell les formule : Chapitre 3 : L'antisémitisme de gauche (1. Idéologie et fonction de l'antisémitisme populaire ; 2. L'antisémitisme plébéien selon Drumont ; 3. L'exploitation politique de l'antisémitisme), Chapitre 5 : Les structures de l'antisémitisme (1. La Ligue antisémitique de France; 2. L'agitation nationaliste et antisémite de la fin du siècle). Cf. Z. Sternhell, La Droite révolutionnaire, op. cit., 1978.

17 Zeev Sternhell, Mario Sznajder et Maia Asheri, Naissance de l'idéologie fasciste, Paris, Fayard, coll. « L'espace du politique », 1989, p. 115-119.

${ }^{18}$ Ibid., p. 13.
} 
Dans $N i$ droite $n i$ gauche, Sternhell repère l'antisémitisme qui anime Marcel Déat, Alfred Fabre-Luce, Gaston Bergery, Jean de Fabrègues et Henri de Man. Cependant, tous ceux parmi les intellectuels dont il dissèque la fascination pour le nazisme ne reprennent pas toujours à leur compte la haine des juifs, tel Robert Aron, Arnaud Dandieu et Emmanuel Mounier en particulier. Il est vrai qu'à confondre le fascisme avec la séduction que celui-ci a pu exercer au-delà de ses chefs et de ses doctrinaires, au-delà des collaborateurs à ses revues et des adhérents et militants à ses organisations, la variable antisémite n'est plus une composante systématique de cette indéniable fascination. Cependant, les frontières sont poreuses car si le centre de gravité fasciste demeure le combat sans merci contre la démocratie libérale, l'acquis en l'absence duquel elle ne serait ni une démocratie ni un régime libéral, reste bel et bien l'émancipation des juifs, leur accès à la citoyenneté, leur intégration juridique et politique, la garantie de leurs droits individuels, de leur liberté de culte et de conscience ${ }^{19}$.

\section{Un itinéraire exemplaire}

L'œuvre de Sternhell forme un bloc constitué de cinq livres auxquels se rattachent plusieurs ouvrages collectifs, des articles de revue et l'autobiographie sous forme d'entretien réalisé en 2015 par Nicolas Weil avec le célèbre historien. Celui-ci passe en revue son itinéraire personnel - de Przemysl en Pologne où il est né en 1935, au ghetto, à Avignon, où il atterrit après-guerre, puis son départ pour Israël en 1951, prenant part à tous ses combats militaires de 1956 à 1982 et à nombre de ses luttes politiques comme membre du parti travailliste et éditorialiste au quotidien Haaretz. Il retrace son parcours professionnel inauguré à l'université hébraïque de Jérusalem, poursuivi à Sciences-po Paris à la fin des années 1960 pour y faire sa thèse, puis aux États-Unis (U.C.L.A., Princeton) où il est régulièrement invité. Couronnement de sa carrière, le prix Israël lui est décerné en 2008 pour l'ensemble de son œuvre et sa contribution à la formation de milliers d'étudiants de l'université hébraïque de Jérusalem où il a enseigné près de quarante ans. Le titre du livre, Histoire et Lumières, définit l'horizon professionnel et intellectuel qu'il s'est fixé comme repère alors que sa recherche a porté sur les hommes qui n'ont eu d'autre passion que de les combattre. C'est peu de dire que Sternhell a résisté à toutes les objections et à toutes les

${ }^{19}$ Zeev Sternhell, Ni droite ni gauche, op. cit., (4 édition), p. 602-603. 
tentations : il ne s'est jamais égaré hors du sentier des Lumières, allant jusqu'à monter au créneau dans les années 1990 pour dénoncer sans concession les séductions dangereuses du post-modernisme prompt à pourfendre les notions de vérité, d'objectivité et d'universalisme en leur substituant les notions fuyantes et frelatées de relativisme et de particularisme ${ }^{20}$. C'est parce qu'il était convaincu de la justesse du grand récit émancipateur qu'il dédia sa vie de chercheur à étudier, non ses défenseurs attitrés, mais ses ennemis jurés, et dont l'entreprise a été un temps couronné de succès pour le malheur de l'humanité. Cette inquiétude sur la fragilité des démocraties allant jusqu'à l'obsession explique pourquoi Sternhell ne s'est jamais interrogé sur l'autre totalitarisme issu, lui, de la Raison. Était-ce parce que le communisme soviétique et stalinien avait comme motivation fondamentale un projet généreux, ce qui n'était guère le cas du nazisme et de son postulat de la race supérieure et de l'espace vital ? Ou bien croyait-il, dans le cas communiste, que ce n'était pas dans les idées de Marx et de ses épigones que gisait son dévoiement potentiel, mais dans la pratique et la contingence qui avait placé Staline à la tête de l'URSS $^{21}$ ? Ce fut, il faut bien le dire, l'angle mort de la recherche menée par Sternhell : il n'en avait que pour les ennemis de la Raison, pas pour ses faux-amis.

Sternhell a été un optimiste inquiet: optimiste, parce que les Lumières préconisent un modèle de constitution politique, d'intelligence du social et de l'individu, qui demeure le seul à poursuivre le triple idéal de liberté et d'égalité et de justice ; inquiet cependant, parce qu'aussi dotées soient-elles pour éclairer l'homme et l'humanité, les Lumières n'en sont pas moins précaires et tremblantes malgré leur prétention à l'universalité. Des forces hostiles ne cessent de les ternir, de les voiler, de faire vaciller la flamme qui les anime dans le but déclaré de les éteindre. Sternhell, l'homme et pas seulement l'historien, est toujours resté tourmenté par le processus historique par lequel une démocratie s'écrase en plein vol. Les turbulences et les tempêtes extérieures forment une première menace ; une autre se situe dans la boite noire qui enregistre les dysfonctionnements antérieurs au décollage. Car s'il n'y a pas de démocratie parfaite, il n'y a pas non plus de démocratie irréversible. Toute sa recherche a procédé de son inquiétude qu'il avait chevillée au

\footnotetext{
${ }^{20}$ Voir Zeev Sternhell, « Bi-zehout Ha-moderniout » [Éloge de la modernité], Politika n48, mars 1993, p. 9-13.

${ }^{21}$ Zeev Sternhell, Histoire et Lumières. Changer le monde par la raison. Entretiens avec Nicolas Weill, Paris, Albin Michel, coll. « itinéraires du savoir », 2014.
} 
corps. «Rien n'est jamais acquis à l'homme », dit le poète, « ni sa force ni sa faiblesse ni son cœur», encore moins la démocratie et la République, aurait pu ajouter Sternhell. Celle-ci a succombé devant les coups portés contre elle par ses adversaires acharnés.

\section{Des Origines du fascisme aux Origines d'Israël}

Cette fragilité intrinsèque a obsédé Sternhell, et c'est sur elle que s'opère une jonction possible entre sa recherche sur le fascisme en Europe et son engagement politique en Israël. On lui a reproché de tirer prétexte de son savoir pour insinuer que les premiers soubresauts de la bête rongent déjà la démocratie israélienne, telle sa déclaration publiée dans Le Monde du 18 février 2018, selon laquelle « en Israël pousse un racisme proche du nazisme à ses débuts $»^{22}$. Si la poursuite de la domination israélienne dans les territoires occupés a pu ne pas freiner, dans un premier temps, l'élan parallèle de démocratisation et de libéralisation des institutions apparu au milieu des années 1990, depuis elle mine le régime démocratique. Certes, la droite israélienne et le sionisme national-religieux, depuis plus de dix ans au pouvoir ne contestent pas le régime parlementaire, mais le tournant illibéral s'accentue : l'indépendance de l'autorité judiciaire est menacée par ceux qui dénoncent sa propension à favoriser le caractère démocratique de l'État d'Israël au détriment de son caractère juif, interprété dans un sens ethno-national exclusif. L'histoire est susceptible de se répéter, redoute-il, même si les moyens et les méthodes diffèrent. Dans le sillage du populisme actuel dont Sternhell a dénoncé les premiers ravages, il suffirait de faire sauter les digues, une par une, à la faveur de telle ou telle opportunité, à commencer par la Cour suprême, la haute fonction publique, puis les media pour terminer.

Cette continuité d'un livre à l'autre, pour finir avec cette somme globale qui retrace deux siècles de réaction antirépublicaine et antirationaliste, est l'indicatif d'une constance et d'une fidélité de l'homme et du chercheur ${ }^{23}$. Cependant, par rapport à cet ensemble

\footnotetext{
22 Il est à noter qu'en l'occurrence Sternhell n'est pas un spécialiste de l'Allemagne nazie, mais a tout au plus établi des distinctions entre fascisme et nazisme.

${ }^{23}$ Nicolas Weill observe avec perspicacité : «Si l'on relit votre Maurice Barrès et le nationalisme français, on peut constater que l'idée qui anime votre ouvrage de 2006, Les Anti-Lumières - la mise en évidence, à travers l'étude du nationalisme, de l'existence d'un puissant courant de pensée réactif au rationalisme du XVIII ${ }^{\mathrm{e}}$ siècle - que vous
} 
cohérent et compact de cinq livres constitutifs d'une bible de l'antifascisme, et sur lesquels il n'a cessé de revenir, multipliant les rééditions, revues et actualisées, de chacun d'eux, il est un ouvrage supplémentaire qui semble, de prime abord, hors-sujet: Aux origines $d^{\prime} I s r a e ̈ l^{24}$. S'il retrace une période de l'Histoire étalée de 1904 à 1940, à peine un peu plus resserrée que celle de ses quatre volumes sur la France de la Troisième République ${ }^{25}$, il ne porte pas sur le fascisme en Europe, mais sur le sionisme socialiste en Palestine. Cette recherche singulière fait fonction d'intrus et présente toutes les apparences d'une exception à la règle que l'autorité incontestée de Sternhell pouvait amplement justifier même s'il n'était assurément pas un spécialiste du sionisme. Lorsqu'on recense les personnages principaux qui se détachent des quatre volumes de Sternhell - Barrès et Doriot, Déat et Drieu, Maurras et Morès, Déroulède et Jouvenel, Drumont et Valois, Vacher de Lapouge et Henri de Man - il va sans dire que les figures qui dominent ce volume à part - David Ben Gourion, Berl Katznelson et Yitzhak Tabenkin ignoraient tout des premiers et n'avaient rien de commun avec eux. Sternhell, qui s'était penché là, une fois n'est pas coutume, sur la famille politique dans laquelle il s'était toujours reconnu, se déclarait sioniste tout comme eux, leur reprochant cependant d'avoir été de piètres socialistes, nullement des fascistes. L'objectif du livre souligné par le titre hébraïque original était d'examiner la prétention de la gauche sioniste depuis le début de l'entreprise menée en Palestine à brandir deux drapeaux, l'un bleu et blanc, l'autre rouge: l'édification d'une nation [binyane ouma - nation-building] et la réforme d'une société [tikkoun hevra]. Sternhell reproche à ces sionistes socialistes non seulement d'avoir sacrifié la construction du socialisme pour se vouer à la construction de l'État, mais en outre, de l'avoir nié, d'avoir continué à se proclamer pour la nation juive et le socialisme alors qu'il n'en a rien été. Entre nationalisme et socialisme, sous-titre de l'ouvrage, le dilemme a été de courte durée : le nationalisme a toujours été prioritaire et le socialisme est resté une façade.

résumez par ce que vous appelez « les Lumières franco-kantiennes », est déjà présent au début de votre parcours... », Histoire et Lumières, op. cit., p. 165.

${ }^{24}$ Zeev Sternhell, Aux origines d'Israël. Entre nationalisme et socialisme, Paris, Fayard, coll. « L'espace du politique », 1997.

${ }^{25} \mathrm{Sa}$ thèse sur Barrès porte sur son nationalisme cristallisé par l'affaire Boulanger et l'affaire Dreyfus, le livre suivant, La Droite révolutionnaire, est sous-titré 1885-1914, Naissance de l'idéologie fasciste, outre la partie consacrée à l'Italie mussolinienne, est consacré au rôle de Georges Sorel dans la gestation du fascisme en France, tandis que Ni droite ni gauche examine la période de l'entre-deux guerres, de 1918 à 1940. 
Pour répondre à notre interrogation et conclure cet hommage à ce grand historien dont la réputation a largement débordé l'enceinte universitaire, nous nous appuierons sur deux arguments complémentaires : l'un tient à une volonté de cohérence ; l'autre procède d'une éthique de travail.

En filigrane de son enquête minutieuse et systématique sur le bouleversement idéologique qui a fait basculer l'Europe des Lumières vers les anti-Lumières de la fin du XIX ${ }^{\mathrm{e}}$ siècle jusqu'à la première moitié du $\mathrm{XX}^{\mathrm{e}}$ siècle, Sternhell n'a jamais caché ou censuré son attachement et sa gratitude pour le modèle d'un individu appuyé sur la Raison. La dernière page des Anti-Lumières est une prière autant qu'un plaidoyer pour préserver l'héritage des Lumières jusque dans le $\mathrm{XXI}^{\mathrm{e}}$ siècle : «La culture des Lumières est une culture critique, pour elle aucun ordre n'est légitime du seul fait qu'il existe. Aucun ordre politique n'est légitime s'il est injuste. [...] Pour éviter à l'homme de sombrer dans un nouvel âge glacé de la résignation, la vision prospective créée par les Lumières d'un individu acteur de son présent, voire de son avenir, reste irremplaçable $»^{26}$. En termes politiques, la cité des Lumières s'est incarnée dans une nation civique fondée sur «la communauté des citoyens ", pour reprendre le titre éponyme du livre de Dominique Schnapper, lesquels dépassent et transcendent leurs attachements familiaux, ethniques et religieux spécifiques par leur quête commune du bien commun ${ }^{27}$. Toutes les variétés d'antirépublicanisme n'ont eu de cesse de substituer à la nation civique la nation ethnique, grâce à laquelle il a été possible, en se fondant sur le sang, la race, la religion, la terre et les morts, d'exclure des citoyens et de les priver de leurs droits. Les juifs ayant été souvent voués à l'exclusion et à la discrimination, jusqu'à la persécution et à l'extermination, le sionisme, pour Sternhell, fut une bouée de sauvetage qui n'a eu de raison d'être que d'avoir fourni à des réfugiés et des apatrides un abri et une citoyenneté. Que le retour se soit fait à Sion, et pas ailleurs, était dans la logique culturelle et politique : s'il était urgent de se rassembler, où le faire sinon dans le foyer d'où a surgi la civilisation juive outre le fait qu'à cette époque, le sort de cette terre n'était pas encore politiquement scellé ? Une place était à prendre et, pour la saisir, il a fallu passer par la guerre, par un conflit à ce jour toujours non résolu, qui a entraîné dans son sillage un nouvel exode, celui des

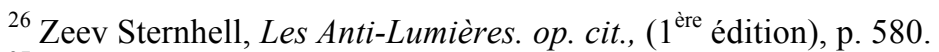

${ }^{27}$ Dominique Schnapper, La Communauté des citoyens. Sur l'idée moderne de nation, Paris, Gallimard, coll. « NRF essais », 1994.
} 
Palestiniens. Sternhell n'ignore pas que le sionisme et l'État d'Israël ont été bâtis sur d'autres fondations que celles de la nation civique. Le sionisme n'est pas un républicanisme, mais un nationalisme culturel. De fait, l'État d'Israël n'a pas généré une nation israélienne, mais des citoyens israéliens qui préservent, au sein de leur communauté respective, leurs attachements ethnoculturels et religieux. Il n'est pas besoin d'être grand clerc ou Sternhell lui-même pour comprendre que si une nation civique, comme la nation française, issue des Lumières et de la Révolution de 1789, n'a pu empêcher que se liguent contre elle des forces pour la renverser, alors, à plus forte raison, une inquiétude analogue a habité Sternhell concernant Israël, et principalement depuis 1967 : une culture politique qui ne s'est pas fondée sur les Lumières n'est-elle pas susceptible de céder plus facilement à « l'irruption de l'irrationnel, la destruction de l'unité du genre humain, une foi absolue devant les capacités de la puissance politique, et donc de l'État, à façonner la société ${ }^{28}$ ? Certes, le judaïsme a posé le premier, et avec vigueur, cette unité du genre humain dont les Lumières ont hérité, ce qui est susceptible de demeurer un garde-fou protecteur, mais il n'est pas inconcevable qu'une partie de la nation juive déforme le sens moral de l'élection pour n'en livrer qu'une version contraire à l'esprit de justice et d'égalité qui l'anime. Aussi, dans un souci de cohérence avec le reste de son œuvre, Sternhell a pensé que le tournant nationaliste pris par Israël depuis plusieurs décennies ne pouvait s'expliquer par les événements fussent-ils la guerre des Six-Jours. Son ethos d'historien l'a conduit à ne pas expliquer cette régression nationaliste sourde aux revendications palestiniennes légitimes à l'autodétermination par la sociologie électorale ou encore par le post-modernisme ou la mondialisation. Fidèle à son approche archéologique qui exclut qu'un tournant soit un phénomène conjoncturel sui generis, Sternhell a estimé que ce cours nouveau plongeait ses racines dans les débuts du mouvement sioniste, «aux origines d'Israël », dans une synthèse entre nationalisme et socialisme, où la part universaliste a été absorbée et dissoute, ainsi qu'il l'a montré sans la moindre indulgence, voire avec un excès de sévérité qui lui a été reproché. Sternhell s'est abstenu de faire peser sur la droite israélienne la responsabilité exclusive de ce désenchantement politique. Il a évité ainsi la tentation de céder à la nostalgie pour ce temps où la gauche travailliste était aux affaires lorsqu'Israël était encore identifié et admiré dans le monde pour l'utopie volontariste du kibboutz. Son interrogation

\footnotetext{
${ }^{28}$ Ibid., p. 580.
} 
convergeait avec celle de Michael Walzer qui s'est lui aussi demandé pourquoi des mouvements nationaux d'émancipation de type progressiste et laïc, comme en Inde, en Algérie et en Israël, se sont essoufflés et traversent une phase de repli identitaire et religieux ${ }^{29}$. Les critiques n'ont pas manqué, telle Anita Shapira qui, partageant avec lui un dépit analogue sur l'État d'Israël aujourd'hui, n'estime pas pour autant que c'est une raison suffisante pour reporter sur le passé tout le poids des errements du présent, jeter le bébé avec l'eau du bain et priver d'une sépulture décente le cadavre travailliste moribond ${ }^{30}$.

On peut également apporter une autre explication à ce livre à part dans la bibliographie de Zeev Sternhell, dont la source est justement la controverse en France autour de son œuvre. Sternhell est intervenu à plusieurs reprises dans la polémique et l'a même intégrée dans les rééditions revues et augmentées de ses livres en réglant ses comptes avec quelques-uns de ses rivaux. La levée de boucliers contre lui, explique-t-il, tient à ce qu'il n'a pas hésité à tendre à l'historiographie française, sinon aux Français dans leur ensemble, un miroir moins glorieux que celui dans lequel les Français se sont regardés et admirés. Sternhell ne pouvait être soupçonné de francophobie. Mais pour être un admirateur ardent et sincère des Lumières et de la Révolution française, il n'a pas renoncé à écorner le roman national, l'image d'Épinal d'une France dans laquelle Vichy n'était qu'une fâcheuse et regrettable parenthèse, mais une parenthèse tout de même, alors qu'il était, aux yeux de Sternhell, préparé par un peu plus d'un demi-siècle de réflexion idéologique et de combat politique. Bref, la figure lumineuse que présente une nation exige, en retour, que lui soit tendu son visage déformé, sa part d'ombre. L'attitude de Sternhell force le respect: il est toujours beaucoup plus courageux d'examiner avec distance et sévérité un roman national retouché et aseptisé que d'en être un lecteur indulgent, passif et, au final, complice.

Comme on avait osé écrire que c'était l'enfant de la Shoah qui prenait là sa revanche - alors que Sternhell fut dans un ghetto en Pologne sans que Vichy y ait la moindre part - il n'est pas improbable qu'il ait souhaité montrer par ce livre qu'il était capable d'examiner Israël d'hier et d'aujourd'hui avec la même sévérité qu'il avait manifestée dans l'étude de l'histoire contemporaine de la France. Il a concrétisé ainsi cette valeur des

\footnotetext{
29 Michael Walzer, The Paradox of Liberation: Secular Revolutions and Religious Counterrevolutions, New Haven and London, Yale University Press, 2015.

30 Anita Shapira, "Kouvlanato shel Sternhell» [Le grief de Sternhell], Yehoudim hadashim, Yehoudim yeshanim [Juifs nouveaux, Juifs anciens], Tel-Aviv, Am Oved, coll. « Sifriat Ofakim », 1995, p. 298.
} 
Lumières, éminentes entre toutes : l'égalité de traitement et ce au nom d'une exigence éthique qui vaut pour tous, individus, nations et États sans distinction ni privilège.

Comment une civilisation bascule-elle dans la barbarie ? C'est là la question. Cette destinée de l'Europe, dont il a éprouvé les effets dans sa chair, Sternhell n'a jamais cru qu'elle était une fatalité. S'il est toujours difficile d'écrire pourquoi il en a été ainsi, au moins Sternhell a-t-il pu montrer l'enchaînement des idées qui a conduit au pire. Le travail d'historien qu'il a accompli dépasse de loin sa contribution au renouvellement de l'historiographie. Sternhell a posé de graves questions qu'il a traitées sans complaisance. Les réponses qu'il a apportées ne valent pas seulement pour comprendre le passé étudié, mais pour éclairer l'avenir, car il n'est jamais trop tard pour changer le cours de l'Histoire par la Raison. À cet égard, Sternhell a été de ces clercs qui n'ont pas trahi.

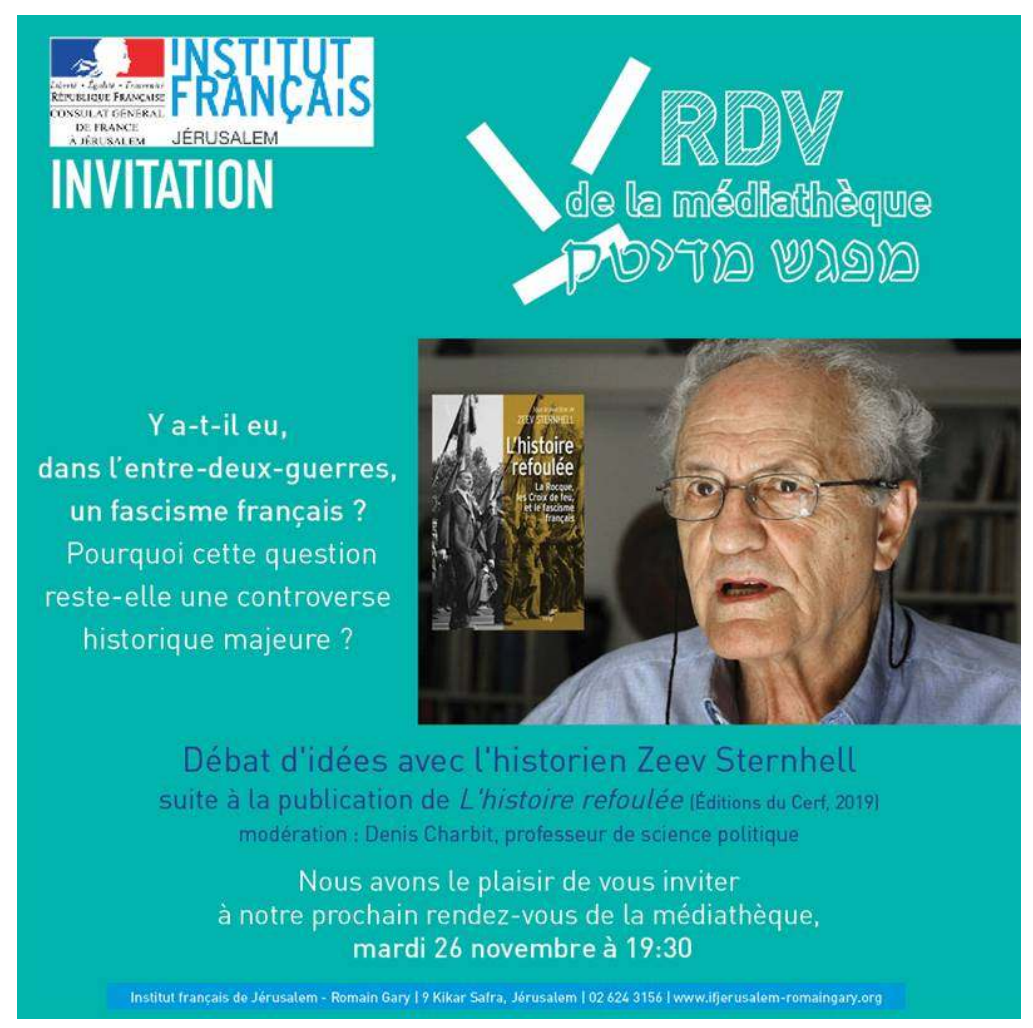

Annonce de la dernière apparition publique de Zeev Sternhell 